Nervenkompressionssyndrome

\section{Kubitaltunnelsyndrom: In-situ-Dekompression versus Verlagerung des Nervus ulnaris}

Staples R et al. Comparative Morbidity of Cubita Tunnel Surgeries: A Prospective Cohort Study. J Hand Surg Am 2018; 43: 207 - 213

Zur chirurgischen Therapie des Kubitaltunnelsyndroms stehen verschiedene Operationsverfahren zur Verfügung, bspw. die offene In-situDekompression und die Verlagerung des Nervus ulnaris. Angesichts des ähnlichen klinisch-neurologischen und funktionellen Ergebnisses beider Techniken haben US-Wissenschaftler untersucht, ob sich die beiden Methoden hinsichtlich der frühen postoperativen Morbidität unterscheiden.

Teilnehmer der an der Abteilung für Orthopädische Chirurgie an der Washington School of Medicine in St. Louis durchgeführten prospektiven Kohortenstudie waren 125 Erwachsene mit einem konservativ therapieresistenten, klinisch und elektrophysiologisch diagnostizierten Kubitaltunnelsyndrom. 47 Patienten wurden mittels In-situ-Dekompression und 78 mittels Verlagerung des Nervus ulnaris (35 subkutane/43 submuskuläre Verlagerung) behandelt. Beide Eingriffe erfolgten über eine Längsinzision über dem Nervenverlauf am posteromedialen Ellenbogen. Die Wahl des Operationsverfahrens lag dabei in der Verantwortung des behandelnden Arztes. Nach Ruhigstellung des operierten Arms über einen Zeitraum von 8 bis 10 Tagen wurde allen Studienteilnehmern eine uneingeschränkte Ellenbogenbeweglichkeit erlaubt. Alle Studienteilnehmer machten vor dem Eingriff anhand einer visuellen Analogskala Angaben zu ihrer Schmerzbelastung und wurden nach der Operation wiederholt zur Stärke der Wundschmerzen befragt. Ferner quantifizierten die Wissenschaftler den Analgetikabedarf, objektivierten die Rate postoperativer Komplikationen (Parästhesien am Olekranon, Hämatome, Infektionen,
Wunddehiszenzen) und erfassten mithilfe des Levine-Katz- sowie des PatientRated Elbow Evaluation-Scores das Vorliegen funktioneller Beeinträchtigungen. Zudem überprüften sie, ob nach einem Zeitraum von mehr als einem Jahr nach der Behandlung ein Revisionseingriff erforderlich geworden war.

Ergebnisse Die mittels In-situ-Dekompression und die mittels Verlagerung des Nervus ulnaris behandelten Patienten unterschieden sich weder hinsichtlich der Alters- und Geschlechtszusammensetzung noch im Hinblick auf die präoperative Schmerzbelastung oder die funktionellen Einschränkungen. Auch das Ausmaß der postoperativen Wundschmerzen war in beiden Gruppen ähnlich, allerdings benötigte ein signifikant größerer Anteil der mittels Nervenverlagerung behandelten Studienteilnehmer 4 bis 8 Wochen nach dem Eingriff Analgetika. Auch der Bedarf an MorphinÄquivalenten war in dieser Studiengruppe signifikant höher. Bezüglich des 1 bis 3 bzw. 4 bis 8 Wochen nach dem Eingriff erhobenen Levine-Katz- sowie des Patient-Rated Elbow Evaluation-Scores waren die mittels Verlagerung des Nervus ulnaris behandelten Patienten gegenüber den Patienten der Dekompressionsgruppe im Nachteil. Zum Zeitpunkt des letzten Follow-ups ließen sich allerdings diesbezüglich keine Unterschiede mehr zwischen den beiden Behandlungsgruppen nachweisen. Olekranon-Parästhesien stellten in beiden Studiengruppen eine häufige Komplikation dar. Bei Studienende waren $11 \%$ der mittels Dekompression und $32 \%$ der mittels Nervenverlagerung behandelten Patienten betroffen. Nach Nervenverlagerung traten 12 Hämatome (15\%) auf. In einem Fall wurde eine chirurgische Ausräumung erforderlich. Alle 5 Hämatome der Dekompressionsgruppe $(11 \%)$ konnten konservativ behandelt werden. Weitere Wundkomplikationen traten nicht auf. Die Auswertung der Patientendokumentationen ergab: Innerhalb von durchschnittlich 2,7 Jahren nach der Operation erfolgten in der Dekompressionsgruppe $6(13 \%)$ und in der Nervenverlagerungsgruppe 2 (3\%) Revisionseingriffe.
FAZIT

Die chirurgische Verlagerung des Nervus ulnaris, so die Autoren, geht im Vergleich zur Dekompression mit einer deutlicheren postoperativen Morbidität einher. Insbesondere muss mit einem höheren Schmerzmittelbedarf, stärkeren funktionellen Einschränkungen innerhalb der ersten Wochen sowie einer höheren Rate persistierender Olekranon-Parästhesien gerechnet werden. Die meisten Komplikationen sind jedoch vorübergehender Natur und bilden sich in der Regel 2 Monate nach dem Eingriff wieder zurück. Revisionseingriffe erfolgten nach In-situ-Dekompression häufiger als nach Verlagerung.

Dr. Judith Lorenz, Künzell 